OPEN ACCESS

Edited by:

Peng He,

Guizhou University, China

Reviewed by:

Nai-Yong Liu,

Southwest Forestry University, China

Joe Hull,

Agricultural Research Service (USDA),

United States

*Correspondence:

Zong-Mao Chen zmchen2006@163.com

Specialty section:

This article was submitted to Invertebrate Physiology, a section of the journal Frontiers in Physiology

Received: 28 August 2017 Accepted: 08 November 2017 Published: 21 November 2017

Citation:

Li Z-Q, Luo Z-X, Cai X-M, Bian L, Xin Z-J, Liu Y, Chu B and Chen Z-M (2017) Chemosensory Gene Families in Ectropis grisescens and Candidates for Detection of Type-II Sex Pheromones. Front. Physiol. 8:953. doi: 10.3389/fphys.2017.00953

\section{Chemosensory Gene Families in Ectropis grisescens and Candidates for Detection of Type-II Sex Pheromones}

\author{
Zhao-Qun Li, Zong-Xiu Luo, Xiao-Ming Cai, Lei Bian, Zhao-Jun Xin, Yan Liu, Bo Chu and \\ Zong-Mao Chen*
}

Key Laboratory of Tea Biology and Resource Utilization, Ministry of Agriculture, Tea Research Institute, Chinese Academy of Agricultural Science, Hangzhou, China

Tea grey geometrid (Ectropis grisescens), a devastating chewing pest in tea plantations throughout China, produces Type-II pheromone components. Little is known about the genes encoding proteins involved in the perception of Type-II sex pheromone components. To investigate the olfaction genes involved in E. grisescens sex pheromones and plant volatiles perception, we sequenced female and male antennae transcriptomes of E. grisescens. After assembly and annotation, we identified 153 candidate chemoreception genes in E. grisescens, including 40 odorant-binding proteins (OBPs), 30 chemosensory proteins (CSPs), 59 odorant receptors (ORs), and 24 ionotropic receptors (IRs). The results of phylogenetic, qPCR, and mRNA abundance analyses suggested that three candidate pheromone-binding proteins (EgriOBP2, 3, and 25), two candidate general odorant-binding proteins (EgriOBP1 and 29), six pheromone receptors (EgriOR24, 25, 28, 31, 37, and 44), and EgriCSP8 may be involved in the detection of Type-II sex pheromone components. Functional investigation by heterologous expression in Xenopus oocytes revealed that EgriOR31 was robustly tuned to the E. grisescens sex pheromone component (Z,Z,Z)-3,6,9-octadecatriene and weakly to the other sex pheromone component (Z,Z)-3,9-6,7-epoxyoctadecadiene. Our results represent a systematic functional analysis of the molecular mechanism of olfaction perception in E. grisescens with an emphasis on gene encoding proteins involved in perception of Type-II sex pheromones, and provide information that will be relevant to other Lepidoptera species.

Keywords: transcriptomic analysis, chemoreception gene, sex pheromone perception, digital gene expression profiling, Ectropis grisescens, Type-II sex pheromone compounds, Xenopus oocytes

\section{BACKGROUND}

In insects, chemical cues are regarded as language and play significant roles in regulating feeding, mating, and ovipositing (Zhou, 2010). Insect antennae, which are covered with several different types of chemosensory sensilla, are the principal chemosensory organs. Olfactory signal transduction starts with the recognition of odor molecules by olfactory receptors, such as odorant receptors (ORs) and ionotropic receptors (IRs) bound to olfactory receptor neuron (ORN) 
dendrites. However, the ORNs that are located within chemosensory sensilla are surrounded by aqueous lymphatic fluid. Thus, water-soluble carriers are required to transport lipophilic compounds through the sensilla lymph. Odorantbinding proteins (OBPs) and chemosensory proteins (CSPs) enhance the solubility of odors and deliver them to the olfactory receptors.

The OBPs of insects comprise $\sim 150$ amino acids and belong to the lipocalins superfamily (Flower, 1996), which comprises carrier proteins folded in the typical $\beta$-barrel structure (Tegoni et al., 2004). Their most striking feature is six highly conserved cysteines paired into three interlocked disulfide bridges (Pelosi et al., 2014). In the Lepidoptera (Gong et al., 2009), OBPs can be classified into pheromone-binding proteins/general odorantbinding proteins (PBPs/GOBPs), antennal binding protein I (ABPI), ABP II, chemical-sense-related lipophilic-ligand-binding protein (CRLBP), Minus-C, and Plus-C OBPs. The PBPs are thought to be involved in pheromone reception processes (Sun et al., 2013; Jin et al., 2014). The GOBPs are encoded by two paralogous genes (GOBP1 and GOBP2) and are thought to be involved in the detection of plant volatiles and sex pheromones (Liu et al., 2015). The CSPs are soluble binding proteins that consist of 100-120 amino acid residues and have four conserved cysteines forming two independent loops (Angeli et al., 1999). Insects CSPs serve varied functions, including chemosensation (González et al., 2009) and development (Maleszka et al., 2007), as well as other processes (Kulmuni and Havukainen, 2013). For example, Sesamia inferens CSP19 and Helicoverpa armigera HarmCSP6 were reported to show high binding affinities for their respective sex pheromone components (Zhang et al., 2014a; Li et al., 2015).

Insect ORs play key roles in detecting odorants and triggering the transduction of chemical signals into electric signals (Spletter and Luo, 2009; Liu C. et al., 2013). Odorant receptor coreceptor (ORco) is one of the most highly conserved $O R$ genes among various insect species (Nakagawa et al., 2012). It interacts with other ligand-specific ORs to form an OR-ORco complex, which functions as a ligand-gated cation channel (Leal, 2012). Pheromone receptors (PRs), a subfamily of ORs, are specifically activated by sex pheromone components and have been widely studied in Lepidopteran insects (Jiang et al., 2014; Zhang et al., 2014b; Chang et al., 2015). The IRs are an important and ancient repertoire of chemosensory receptors involved in olfaction (Benton et al., 2009) and gustation (Zhang et al., 2013). Previous studies have revealed that IRs also need a coreceptor (Benton et al., 2009). The proteins IR8a and IR25a are antennal IR coreceptors that are expressed at higher levels than other IRs in Drosophila and Chilo suppressalis (Rytz et al., 2013; Cao et al., 2014).

Tea grey geometrid, Ectropis grisescens, is a devastating chewing pest distributed in tea plantations throughout China. The sex pheromone components of E. grisescens have been characterized as (Z,Z,Z)-3,6,9-octadecatriene (Z3Z6Z9-18:Hy) and (Z,Z)-3,9-6,7-epoxyoctadecadiene (Z3Z9-6,7-epo-18:Hy) (Ma et al., 2016). Moth sex pheromone components can be divided into three types according to their structure: Type-I, Type-II, and miscellaneous type with proportions of 75,15 , and $10 \%$, respectively (Ando et al., 2004). Type-I sex pheromone components comprise $\mathrm{C}_{10}-\mathrm{C}_{18}$ straight chain unsaturated alcohols, aldehydes, or acetate esters; and Type-II sex pheromone components consist of $\mathrm{C}_{17}-\mathrm{C}_{23}$ straight chains with 1-3 cis double bonds separated by methylene groups (Millar, 2000; Ando et al., 2004). Therefore, E. grisescens produces Type-II sex pheromone components. Most studies on the sex pheromone perception mechanism in Lepidopteran insects have focused on Type-I pheromone components (Jiang et al., 2014; Zhang et al., 2014b; Chang et al., 2015). Comparatively, little is known about Type-II pheromone components (Zhang et al., 2016). It is acknowledged and accepted that olfaction perception plays crucial roles in the chemical detection of $E$. grisescens (Sun et al., 2014; Ma et al., 2016). Thus, analysis of its olfactory molecular mechanism may identify targets for pest control. However, little is known about the molecular mechanisms regulating E. grisescens olfaction because of the paucity of sequence data for olfaction genes from E. grisescens. Therefore, to obtain such data as the primary step for exploring the olfaction mechanism, we constructed cDNA libraries of female and male antennae in E. grisescens and conducted several analyses to identify olfactory-related genes.

\section{RESULTS}

\section{Overview of Antennae Transcriptomes}

The transcriptomes of female antennae (FA) and male antennae (MA) of E. grisescens were sequenced with two independent biological replicates. About 45.72 (FA-1), 46.51 (FA-2), 42.15 (MA-1), and 49.97 (MA-2) million raw reads were obtained for each transcriptome. The datasets of transcriptomes during the current study are available in the NCBI SRA database (http://trace.ncbi.nlm.nih.gov/Traces/sra/, accession numbers: SRR6004297-SRR6004301). After filtering and assembling, 114,595 transcripts were generated with an $\mathrm{N}_{50}$ length of 1,715 nt (Table 1). For annotation, we combined the female- and maleantennal transcriptomes of E. grisescens and searched against the non-redundant (NR) database by BLASTX with a cut-off e-value of $10^{-5}$. The best match percentage $(40.2 \%)$ was with Bombyx mori sequences, followed by sequences from Plutella xylostella (16.7\%), Danaus plexippus (16.6\%), Acyrthosiphon pisum (1.5\%), and Papilio xuthus (1.4\%) (Figure 1A). Gene ontology (GO) annotation was used to classify the transcripts into functional categories.

Among the distinct transcripts, 32,710 (28.54\%) corresponded to at least one GO term.

Within the molecular function category, most transcripts were involved in binding (e.g., nucleotide-, ion-, and odorant-binding) and catalytic activity (e.g., hydrolase and oxidoreductase) (Figure 1B).

\section{Identification of $E$. grisescens OBP/CSP/OR/IR Genes and Sequence Analyses}

Sequence annotation led to the identification of 40 candidate EgriOBPs in the E. grisescens antennae transcriptome (File S1). 
TABLE 1 | Ectropis grisescens antennal transcriptome assembly and annotation.

\begin{tabular}{|c|c|c|c|c|}
\hline & FA-1 & FA-2 & MA-1 & MA-2 \\
\hline Total number of raw reads & $45,722,672$ & $46,510,102$ & $42,151,920$ & $49,965,856$ \\
\hline Total number of clean reads & $43,871,882$ & $44,413,250$ & $40,327,306$ & $47,906,526$ \\
\hline Clean bases & $6.58 \mathrm{~Gb}$ & $6.66 \mathrm{~Gb}$ & $6.05 \mathrm{~Gb}$ & $7.19 \mathrm{~Gb}$ \\
\hline Total number Clean Nucleotides (nt) & $6.58 \mathrm{~Gb}$ & $6.66 \mathrm{~Gb}$ & $6.05 \mathrm{~Gb}$ & $7.19 \mathrm{~Gb}$ \\
\hline $\mathrm{Q}_{20}$ percentage & $97.51 \%$ & $97.54 \%$ & $97.26 \%$ & $97.53 \%$ \\
\hline GC percentage & $40.19 \%$ & $40.06 \%$ & $39.68 \%$ & $40.27 \%$ \\
\hline Total number of transcripts & \multicolumn{4}{|c|}{114,595} \\
\hline $\mathrm{N}_{50}(\mathrm{nt})$ & \multicolumn{4}{|c|}{1715} \\
\hline Percentage of transcripts annotated by NCBI NR database & \multicolumn{4}{|c|}{$35.78 \%$} \\
\hline Percentage of transcripts annotated by Swiss-prot database & \multicolumn{4}{|c|}{$24.77 \%$} \\
\hline Percentage of transcripts annotated by PFAM database & \multicolumn{4}{|c|}{$28.34 \%$} \\
\hline Percentage of transcripts annotated by KOG database & \multicolumn{4}{|c|}{$17.8 \%$} \\
\hline Percentage of transcripts annotated by GO database & \multicolumn{4}{|c|}{$28.54 \%$} \\
\hline
\end{tabular}

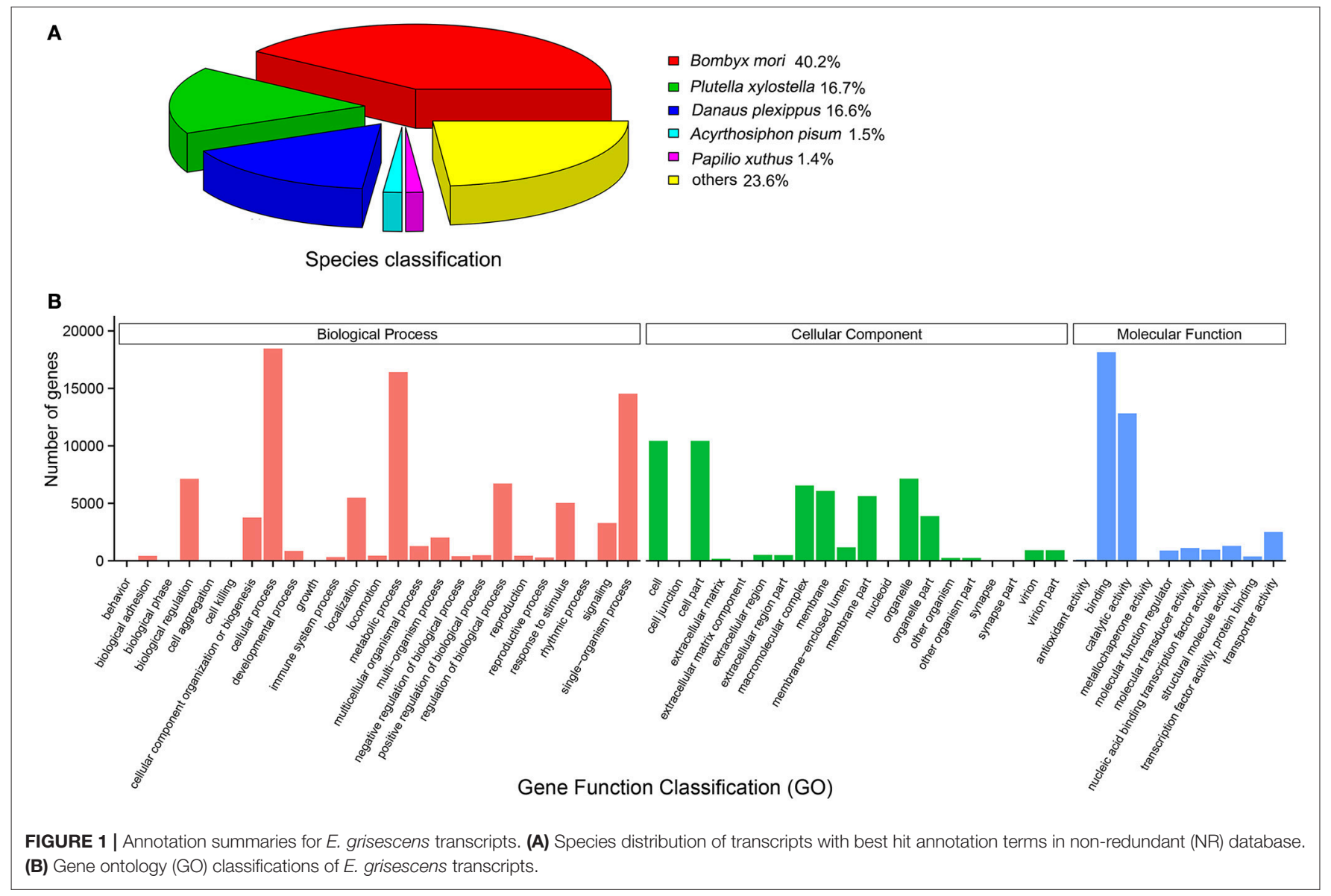

Sequence analyses showed that 34 of them were full-length EgriOBP genes, and 31 had a predicted signal peptide (Figure 2A). EgriOBP13, 14, 15, 32, 37, and 39 contained four conserved cysteines but lacked the conserved cysteines C2 and C5. EgriOBP4, 5, 6, 7, 28, 35, and 40 contained more than six conserved cysteines. EgriOBP8 contained five conserved cysteines but lacked the conserved cysteine C2. All of the E. grisescens classic OBPs present the C-pattern common to Lepidoptera, C1- $\mathrm{X}_{25}-30-\mathrm{C} 2-\mathrm{X} 3-\mathrm{C} 3-\mathrm{X}_{36-42}-\mathrm{C} 4-\mathrm{X}_{8-14}-\mathrm{C} 5-\mathrm{X}_{8}$ C6. A total of 30 CSP genes were identified in E. grisescens antennae, 26 of which contained a full-length open reading frame (ORF), a signal peptide, and four conserved cysteine residues (Figure 2B). All of the E. grisescens CSPs have the C-pattern of Lepidoptera, $\mathrm{C} 1-\mathrm{X}_{6-8}-\mathrm{C} 2-\mathrm{X}_{18}-\mathrm{C} 3-\mathrm{X}_{2}-\mathrm{C} 4$. By homology analysis, 


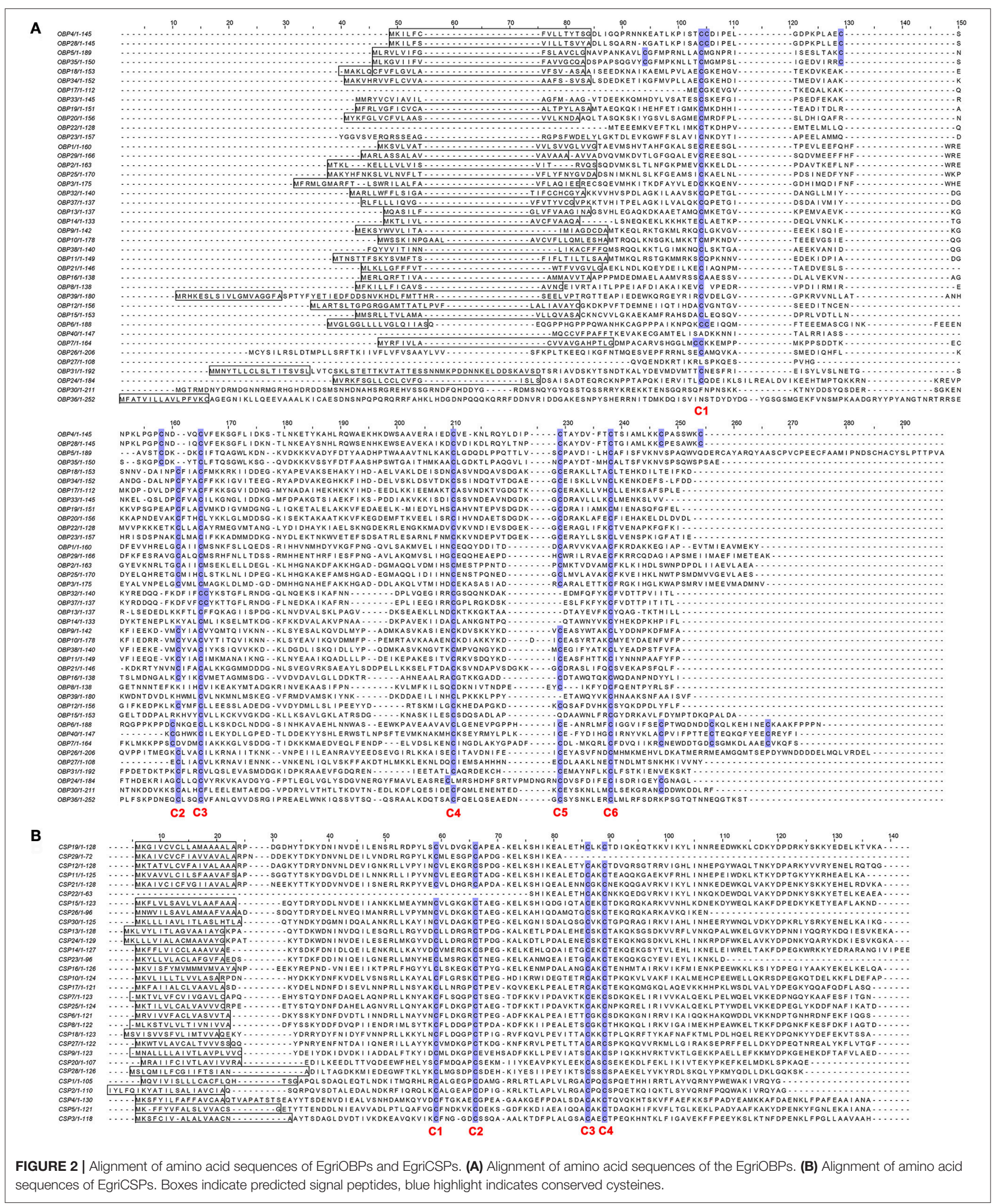


we identified 59 candidate EgriORs in E. grisescens antennae. Sequence analyses revealed that 45 (EgriOR1-44 and EgriORco) of the 59 sequences had an intact ORF with characteristics typical of insect $O R$ genes (full-length ORFs of about 1,200 bp). In total, we identified 24 EgrilRs from E. grisescens antennal transcriptomes, and 16 of them contained intact ORFs.

\section{Phylogenetic Analyses}

To further investigate the function of E. grisescens OBP/CSP/OR/ $I R$ genes, phylogenetic trees were constructed using sequences of typical OBP/CSP/OR/IRs from other insect species for which the whole genome or transcriptome data were available. In the resulting phylogenetic tree, we observed four well-supported groups; PBP, GOBP, Plus-C OBP, and Minus-C OBP (Figure 3). Three EgriOBPs (EgriOBP2, 3, and 25) were grouped in the PBP clade with another Lepidoptera PBP. The orthologs EgriOBP1 and 29 were in the GOBP group. EgriOBP4, 5, 6, 7, 28, 35, and 40 were distributed in the Plus-C OBP group, and EgriOBP8, 13, 14, $15,32,37$, and 39 were distributed in the Minus-C OBP group. In the CSP phylogenetic tree, EgriCSP8 was grouped into the same clade as HarmCSP6 (Figure 4).

The EgriORs were distributed among the orthologous groups in the OR phylogenetic tree (Figure 5). EgriORco was strongly associated with ObruORco, HvirORco, and BmorOR2 with high bootstrap support. EgriOR25, 28, and ObruOR1 were grouped with B. mori, H. armigera, Helicoverpa assulta, and Heliothis virescens PRs, which are known to be receptors for TypeI pheromones. EgriOR24, 31, 37, and 44 were independently grouped without any orthologs of other Lepidoptera insects. In the IR phylogenetic tree (Figure 6), 11 EgriIRs (Egri3, 6, 8, 11, $12,13,14,16,18$, and 24) were clustered with the presumed "antennal" orthologs IR64a, IR21a, IR31a, IR68a, IR75d, IR76b, IR93a, IR60a, and IR40a. Two EgriIRs, EgriIR10, and EgriIR21, were respectively distributed in the IR8a and IR25a groups, which are co-receptors. EgriIR1 was grouped with NMDA iGluRs (N-methyl-d-aspartate ionotropic receptors).

\section{Tissue Expression Profile and mRNA Abundance of $E$. grisescens OBP/CSP/OR/IR Genes}

We further characterized the tissue expression pattern and abundance of $E$. grisescens $O B P / C S P / O R / I R$ genes in the antennae by qPCR and by evaluating the RPKM (reads per kilobase per million mapped reads) values. The qPCR results indicated that 24 EgriOBPs were uniquely or more strongly expressed in female and male antennae, except for EgriOBP4, 7, 8, 15, 21, $22,23,24,27,28,35,37,39$, and 40 (Figure 7A). Of these 24 EgriOBPs showing antenna-biased expression, EgriOBP2, 3, 9, 12 , and 25 showed significantly higher transcript levels in male antennae than in female antennae (9.8-, 10.3-, 9.0-, 7.8-, and 12.8-fold higher RPKM values, respectively, in male antennae than in female ones). Of the five male antenna-biased EgriOBPs, EgriOBP2 and 3 were much more abundant in the antennae transcriptome. EgriOBP7, 13, 21, and 33 transcripts were abundant mainly in female and male proboscises. Compared with EgriOBPs, EoblCSPs showed wider and more diverse expression patterns (Figure 7B). The transcript levels of EgriCSP5, 8, 13, 14, 15,16 , and 17 were markedly higher in the E. grisescens antennae transcriptomes than in the transcriptomes of other tissues. Of these seven EgriCSPs that were abundant in the antennae, EgriCSP8 was expressed at higher levels in male antennae than in female ones. The abundance of EgriCSP 21 and 25 transcripts was markedly higher in female antennae than in male ones. The abundance levels of EgriCSP5, 15, and 17 were also higher in female antennae than in male ones, but the differences were not significant.

Analyses of the expression profile of EgriORs showed that these EgriOR genes were uniquely or more strongly expressed in antennae than in other tissues (Figure 8A). Among the ORs, including EgriORco, EgriOR28 and 37 showed the highest expression levels in antennae. Five EgriORs (EgriOR24, 28, 37, 44 ) were predominantly expressed in male antennae, with RPKM values in male antennae being 51.6-, 29.6-, 20.9-, and 72.7fold that of their respective counterparts in female antennae. EgriOR31 was uniquely expressed in male antennae. Analyses of the expression patterns of EgriIR genes indicated that EgriIR8, $10,11,12,16,21$, and 24 were highly expressed in the antennae (Figure 8B). Of these eight EgriIRs, EgriIR10, 21, and 24 showed relatively high RPKM values in female and male transcriptomes.

\section{Functional Study of EgriOR31}

The Xenopus oocytes and two-electrode voltage clamping recording system were used to characterize the function of the EgriOR1 and 31, by co-expressing with the corresponding receptor EgriORco. The results showed that oocytes coexpressing EgriOR31 and EgriORco robustly responded to the triene Z3Z9-6,7-epo-18:Hy, but less so to Z3Z6Z9-18:Hy. The oocytes co-expressing EgriOR1 and EgriORco showed no responses (Figure 9).

\section{DISCUSSION}

Chemical cues, including sex pheromones and host plant volatiles, drive several aspects of insect behavior, such as mating, feeding, and selection of oviposition sites. Sex pheromoneinduced behaviors play crucial roles in insect reproduction. The sex pheromone components of E. grisescens are Z3Z6Z918:Hy and Z3Z9-6,7-epo-18:Hy (Ma et al., 2016), both of which are Type-II compounds. However, little is known about the molecular mechanisms regulating E. grisescens olfaction. Therefore, we analyzed the antennal transcriptomes of female and male E. grisescens to identify genes involved in the perception of sex pheromones and host plant volatiles. In our study, we sequenced E. grisescens female and male antennal transcriptomes, with two independent biological replicates. A total of 26.48 $\mathrm{Gb}$ of antennae transcriptome data was obtained. Sequence assembly yielded 114,595 transcripts from E. grisescens antennal transcriptomes. After annotation, we identified 153 candidate chemosensory genes (40 EgriOBPs, 30 EgriCSPs, 59 EgriORs, and 24 EgriIRs) in E. grisescens (File S1).

Insect PBPs represent a sub-class of OBPs that are specifically dedicated to the detection of sex pheromones (Zhou, 2010). 


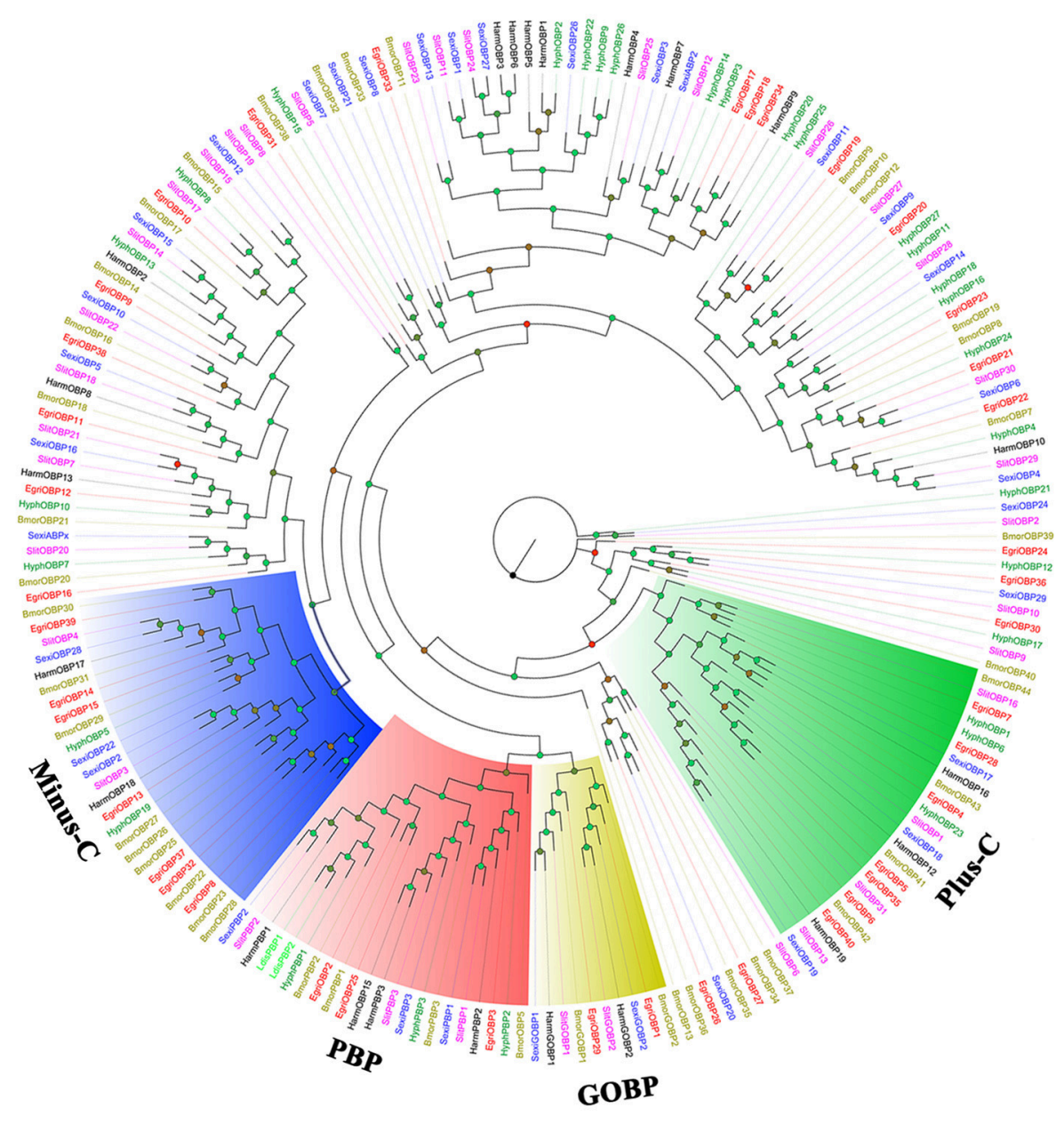

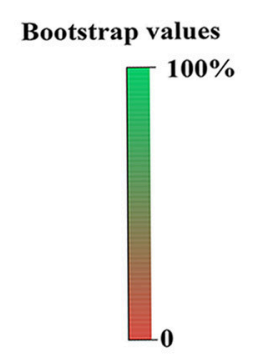

$\overline{3.0}$

Egri, Ectropis grisescens Bmor, Bombyx mori Harm, Helicoverpa armigera Hyph, Hyphantria cunea Sexi, Spodoptera exigua Slit, Spodoptera litura Ldis, Lymantria dispar

FIGURE 3 | Phylogenetic analysis of EgriOBPs with other typical insect OBPs. Phylogenetic tree was constructed in PhyML3.0 using maximum likelihood method.

EgriOBP2 and 3 were the two most abundant EgriOBPs in the antennal transcriptome with $\sim 10$-fold higher RPKM values in male antennae than in female ones. EgriOBP25 showed a relatively high RPKM value in male antennae. The phylogenetic tree showed that EgriOBP2, 3, and 25 were distributed in the PBP group with PBPs from S. inferens (Jin et al., 2014), Spodoptera exigua (Liu et al., 2014), Spodoptera litura (Liu N. Y. et al., 2013), H. armigera (Dong et al., 2017), and Hlyphantria cunea (Sanes and Plettner, 2016). That is, EgriOBP2, 3, and 25 were expressed at higher levels in male antennae than in female ones, they were more abundant than other OBPs in male antennae, and they showed homology to other insect PBPs that are known to function in sex pheromone binding. Therefore, EgriOBP2, 3 and 25 may encode PBPs for TypeII pheromone components. Aside from EgriOBP2, 3, and 25, EgriOBP9 and 12 also showed significantly higher expression in male antennae than in female ones, with relatively high RPKM values in male antennae. However, they were not homologous to PBPs of other Lepidoptera insects that produce Type-I pheromone components. Further research is required to clarify the roles of EgriOBP9 and 12 in sex pheromone perception in E. grisescens.

Another key sub-class of OBPs, the GOBPs, are known to be sensitive to both sex pheromones and plant volatiles (Liu et al., 2015). EgriOBP1 and 29 were distributed in GOBP clade. Of them, EgriOBP1 was grouped in the GOBP2 sub-classed with S. litura (Liu et al., 2015), B. mori (Zhou et al., 2009), and S. exigua (Liu et al., 2014) GOBP2 which could strongly bind sex pheromones. Consequently, we speculate that EgriOBP1 may be involved in the binding of sex pheromone components in E. grisescens.

Based on their cysteine motifs, inset OBPs can be classified into several groups: classical OBPs (with six conserved cysteines), Minus-C (with four conserved cysteines) (Forêt and Maleszka, 2006), Plus-C OBPs (with more than six conserved cysteines) (Hekmat-Scafe et al., 2002; Zhou et al., 2004), and dimer OBPs (which contain two amino acid sequence domains) (Hekmat-Scafe et al., 2002). Several Minus-C OBPs (H. armigera HarmOBP17 and 18 and Apis mellifera OBP 14) show high binding affinities to plant volatiles (Spinelli et al., 2012; 


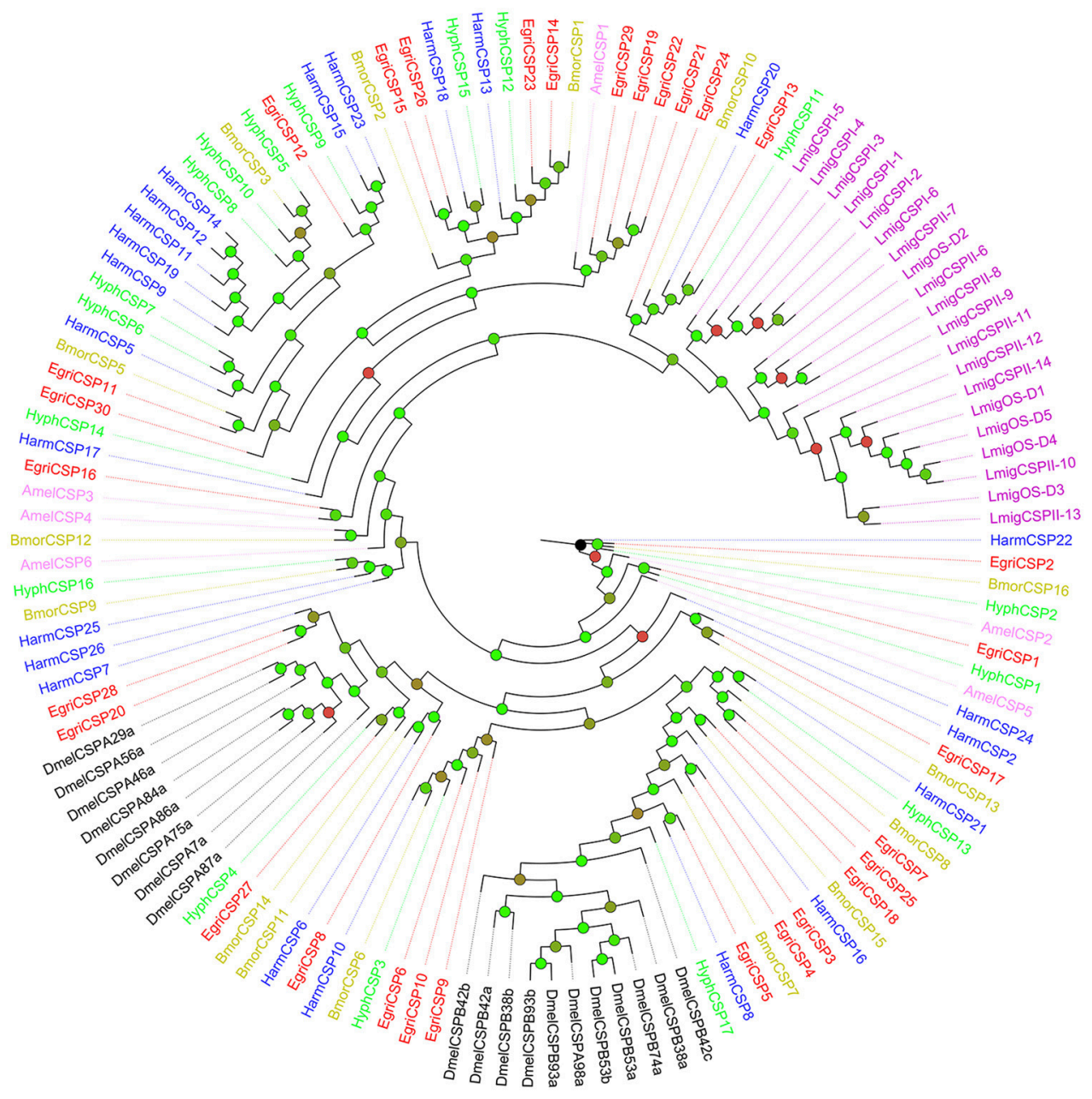

Bootstrap values

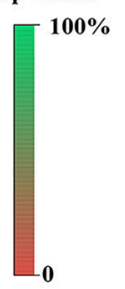

$\overline{3.0}$

Egri, Ectropis grisescens

Bmor, Bombyx mori

Amel, Apis mellifera

Lmig, Locusta migratoria

Dmel, Drosophila melanogaster

Hyph, Hyphantria cunea

Harm, Helicoverpa armigera

FIGURE 4 | Phylogenetic analysis of EgriCSPs with other typical insect CSPs. Phylogenetic tree was constructed in PhyML3.0 using maximum likelihood method.

Li et al., 2013). In our study, EgriOBP14 and EgriOBP13 were distributed in the same cluster with HarmOBP17 and HarmOBP18, respectively. Thus, these two EgriOBPs might have similar functions to HarmOBP17 and 18, and play roles in plant volatiles perception. EgriOBP22 was associated with $H$. armigera HarmOBP10, which is expressed at high levels in both antennae and seminal fluid and may function as a carrier of oviposition deterrents (Sun et al., 2012).

Insect PRs, a key sub-class of ORs, are responsible for detecting sex pheromone components in the Lepidoptera (Jiang et al., 2014; Zhang et al., 2014b; Chang et al., 2015). Due to the lack of data for PRs for Type-II pheromone components, we constructed the phylogenetic tree using PRs for Type-I pheromone components. Out of 59 EgriORs, two (EgriOR25 and 28) were grouped in the PR clade with PRs for TypeI sex pheromone components. Among them, EgriOR28 was predominantly expressed in male antennae, with an RPKM value 29.6-fold higher in male antennae than in female antennae. EgriOR28 was the second most abundant OR in antennae. Therefore, these two EgriORs, particularly EgriOR28, may encode PRs for Type-II sex pheromone components. Generally, Lepidoptera insects have about 5 6 PRs; for example, six PRs were identified in both $H$. armigera (Liu et al., 2012) and C. suppressalis (Cao et al., 2014). On the other hand, Geometroidea species that produce Type-II sex pheromones, including E. grisescens, are more highly evolved than species that produce Type-I sex pheromones. Therefore, we speculate that another EgriORs involved in the detection of sex pheromones in E. grisescens might be distributed in a group separate from that containing PRs for Type-I sex pheromone components.

In fact, four EgriORs (EgriOR24, 31, 37, and 44) formed an independent group in the phylogenetic analysis. In addition, all four of these EgriORs showed higher abundance in male antennae than in female ones (RPKM values in the male antennae being 51.6-, 29.6-, 20.9-, and 72.7-fold that of their respective counterparts in female antennae). Among these four male antenna-biased EgriORs, EgriOR37 was the most abundant EgriOR in antennal transcriptomes, and EgriOR24 and 31 showed relatively high RPKM values in male antennae. 


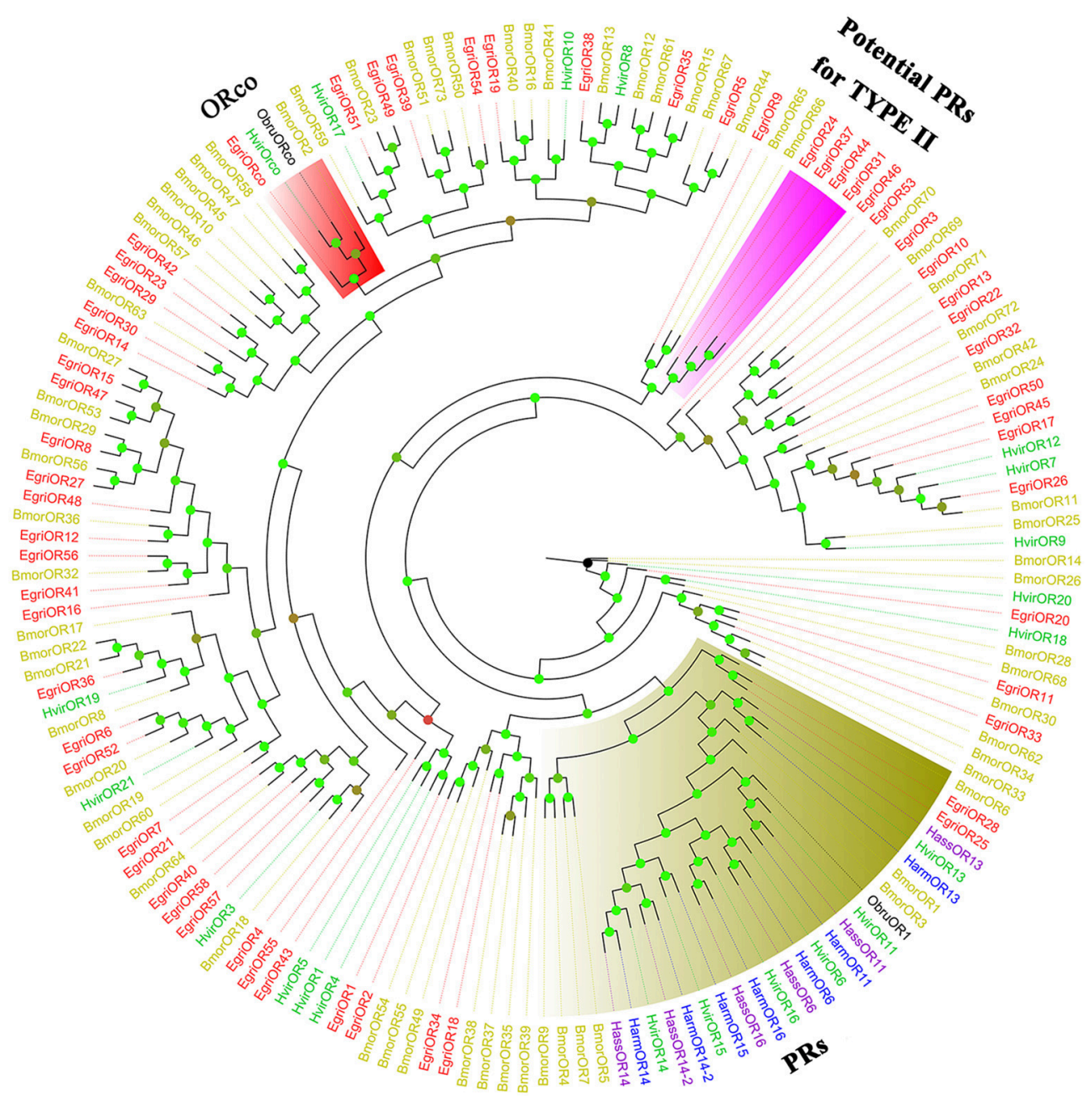

Bootstrap values

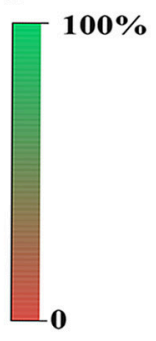

$\overline{\mathbf{3 . 0}}$

Egri, Ectropis grisescens Bmor, Bombyx mori Harm, Helicoverpa armigera Hass, Helicoverpa assulta Hvir, Heliothis virescens

Obru, Operophtera brumata

FIGURE 5 | Phylogenetic analysis of EgriORs with other typical insect ORs. Phylogenetic tree was constructed in PhyML3.0 using maximum likelihood method.

Consequently, it is conceivable that EgriOR24, 31, 37, and 44 might be potential PRs for Type-II sex pheromone components. To test this above hypothesis, we respectively co-expressed EgriOR31 and EgriOR1 (an EgriOR that sorted to a different phylogenetic clade with EgriOR24, 31, 37, and 44) with the corresponding co-receptor EgriORco, and tested it against the sex pheromone of E. grisescens. The results showed that EgriOR31 and EgriORco robustly responded to Z3Z9-6,7-epo18:Hy and weakly to Z3Z6Z9-18:Hy. However, EgriOR1 and EgriORco showed no responses. This result indicated that these four male antennae abundant EgriORs (EgriOR24, 31, 37, and 44) which formed an independent group in the phylogenetic analysis might also be potential PRs for Type-II sex pheromone components.

The CSPs and IRs are known to be involved in insect odorant reception. In the CSP phylogenetic tree, EgriCSP8 grouped in the same clade as HarmCSP6, which is responsible for the perception of sex pheromones (Li et al., 2015). In addition, EgriCSP8 showed a male antenna-biased expression pattern with a relatively high
RPKM value in male antennae, suggesting that EgriCSP8 plays a role in E. grisescens sex pheromone detection. Consistent with the roles of IRs in olfaction, most of the EgriIRs were clustered with "antennal" orthologs and displayed high expression levels in olfactory tissues. The IRs are known to detect acids, amines, and other odorants that are not sensed by ORs (Benton et al., 2009; Ai et al., 2010, 2013). Of the 24 EgriIRs, EgriIR10, 21, and 24, which was grouped in the antennal IR group, showed relatively high RPKM values in female and male transcriptomes. These results indicated that it might play key roles in olfaction, especially in male E. grisescens.

In conclusion, we sequenced the female and male antennae transcriptomes of E. grisescens to identify the genes involved in chemoreception, with an emphasis on genes encoding proteins involved in the perception of Type-II sex pheromone components. The results of phylogenetic, gene expression, and transcript abundance analyses indicate that a number of EgriOBPs, EgriORs, and EgriCSPs with male antenna-biased expression could be involved in sex pheromone detection. In 

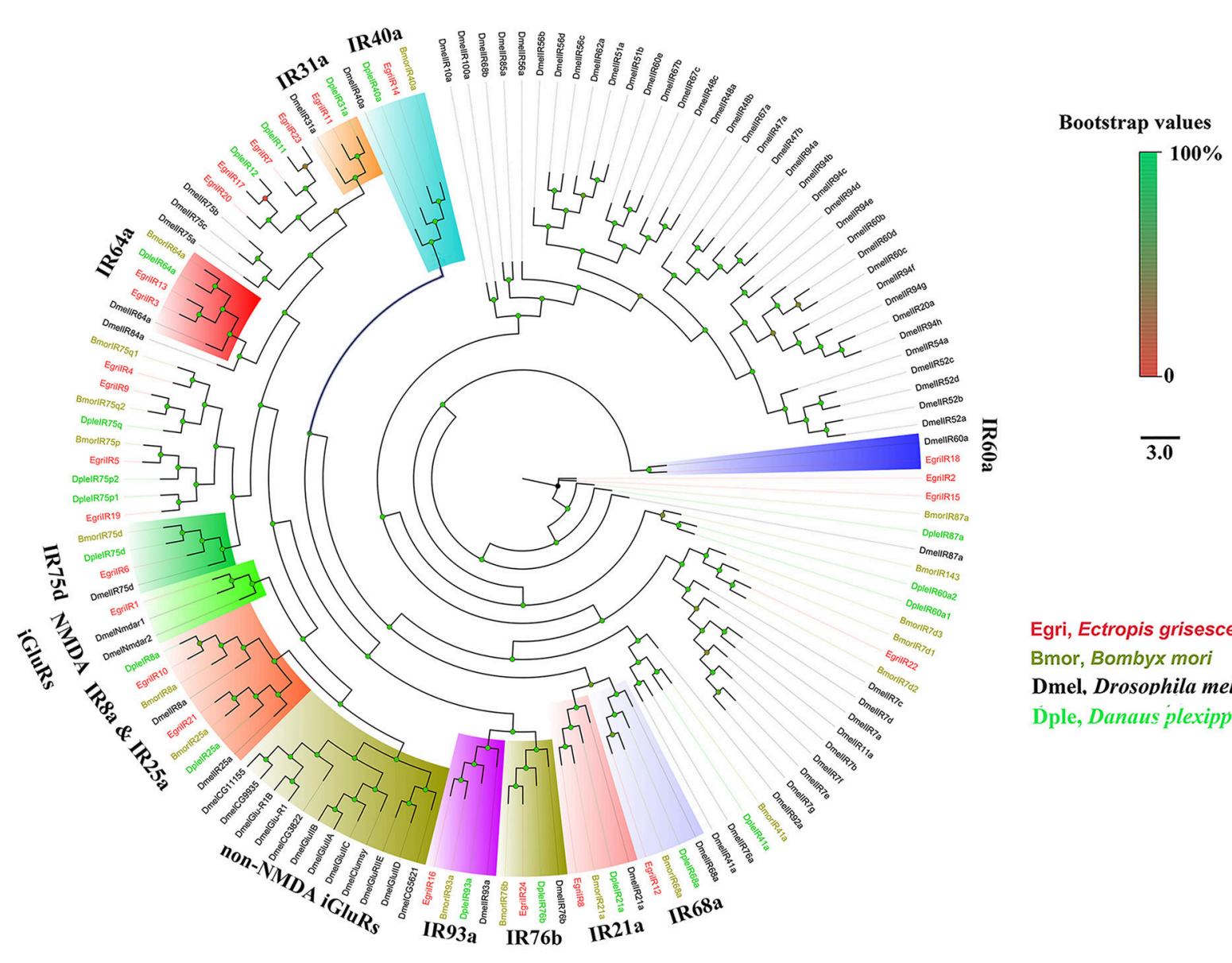

Egri, Ectropis grisescens Bmor, Bombyx mori

Dmel, Drosophila melanogaster D́ple, Danaus plexippus

FIGURE 6 | Phylogenetic analysis of EgrilRs with other typical insect IRs. Phylogenetic tree was constructed in PhyML3.0 using maximum likelihood method.

particular, EgriOR24, 31, 37, and 44 might be potential PRs for Type-II sex pheromone components. Functional investigation revealed that EgriOR31 was tuned to the E. grisescens sex pheromone components.

\section{MATERIALS AND METHODS}

\section{Insect Rearing and Tissue Collection}

Individuals of E. grisescens were originally collected from the Experimental Tea Plantation of the Tea Research Institute, Chinese Academy of Agricultural Sciences (Hangzhou, Zhejiang, China). Experimental insects were reared on fresh tea shoots in enclosed nylon mesh cages $(70 \times 70 \times 70 \mathrm{~cm})$ and kept in a climate-controlled room at $25 \pm 1^{\circ} \mathrm{C}$ and $70 \pm 5 \%$ relative humidity under a 14 -h light: 10 -h dark photoperiod. After pupation, male and female pupae were kept separately in cages for eclosion. After emergence, adult moths were supplied with $10 \%$ honey solution. For transcriptome sequencing, 100 female and 100 male antennae were collected from 2-day-old virgin insects, with two replicates. Female and male antennae, heads, thoraxes, thoraxes, legs, wings, proboscises, and pheromone glands were collected from 3-day-old virgin insects for qRT-PCR analyses. These tissues were immediately frozen and stored at $-80^{\circ} \mathrm{C}$ until RNA isolation.

\section{cDNA Library Preparation and Illumina Sequencing of Transcriptomes}

Total RNA was extracted from female and male antennae using TRIzol reagent (Invitrogen, Carlsbad, CA, USA). Quality of the RNA were assessed by agarose gel electrophoresis, Nanodrop (Thermo), and Agilent 2100. The sampling quality satisfy the requirements of cDNA libraries construction. The cDNA library construction and Illumina sequencing were performed at Novogene Bioinformatics Technology Co., Ltd. (Beijing, China). Briefly, mRNAs were isolated from $5 \mu \mathrm{g}$ pooled total RNA using oligo (dT) magnetic beads and fragmented into short fragments in the presence of divalent cations in fragmentation buffer at $94^{\circ} \mathrm{C}$ for $5 \mathrm{~min}$. Using these short fragments as templates, random hexamer primers were used to synthesize first-strand cDNA. Second-strand cDNA was generated using RNase $\mathrm{H}$, and DNA polymerase I. After end repair and adaptor ligation, short sequences were amplified by PCR and purified with a QIAquick ${ }^{\circledR}$ PCR purification kit (Qiagen, Venlo, The Netherlands), and sequenced on the HiSeq ${ }^{\mathrm{TM}} 2500$ platform (San Diego, CA, USA). 

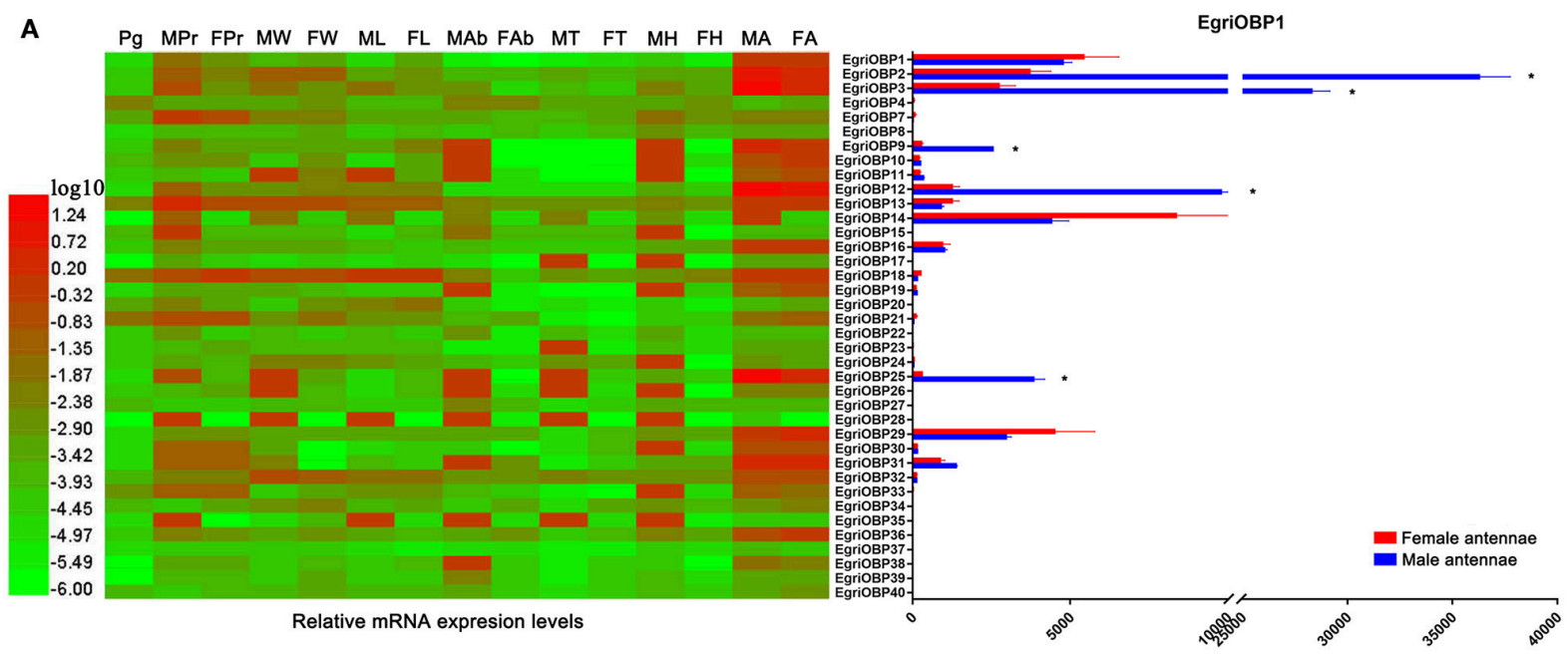

B 2 Pg MPr FPr MW FW ML FL MAb FAb MT FT MH FH MA FA
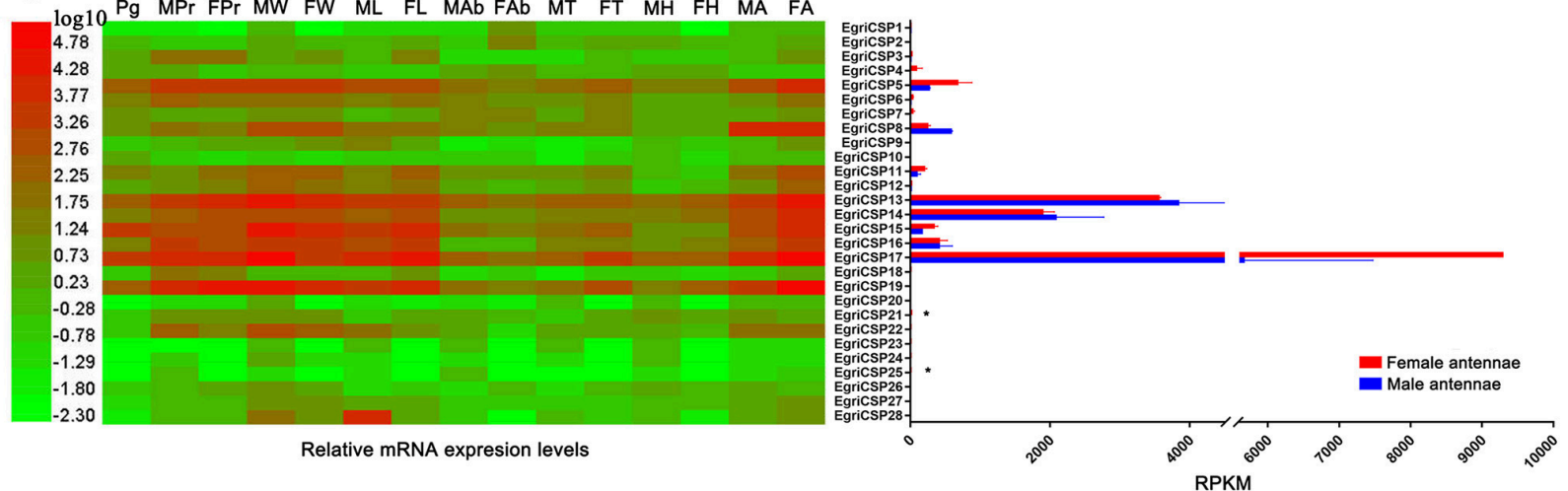

FIGURE 7 | Tissue expression profiles and abundance of selected EgriOBP and EgriCSP genes in antennae based on relative mRNA quantity and RPKM values. Heat map illustrates $\log _{10}$-transformated mRNA expression levels of EgriOBP and EgriCSP in different tissues. Histogram shows RPKM values of EgriOBP and EgriCSP. (A) Tissue expression profile and RPKM values of selected EgriOBP genes. (B) Tissue expression profile and RPKM values of selected EgriCSP genes. FA, female antennae; MA, male antennae; FH, female head without antennae; MH, male head without antennae; FT, female thorax; MT, male thorax; FAb, female abdomen without pheromone glad; MAb, male abdomen; FL, female legs; ML, male legs; FW, female wings; MW, male wings; FPr, female proboscis; MPr, male proboscis; Pg, pheromone gland. ${ }^{*} P$-value $<0.05$.

\section{De Novo Assembly of Short Reads and Functional Annotation}

Transcriptome de novo assembly was carried out with the shortread assembly program Trinity (r20140413p1) (Grabherr et al., 2011) based on the paired-end reads with default settings. Transcripts longer than 150 bp were first aligned by BLASTX to protein databases (NR, Swiss-Prot, KEGG, and COG; $e$-value < $10^{-5}$ ) to retrieve proteins with the highest sequence similarity to the unigenes along with their protein functional annotations. We then used the Blast2GO (Conesa et al., 2005) for GO annotation of the transcripts and WEGO software (Ye et al., 2006) to plot the $\mathrm{GO}$ annotation results.

\section{Analysis of Transcript Expression in the Antennal Transcriptomes}

Transcript abundance was calculated by the RPKM (reads per kilobase per million mapped reads) method (Mortazavi et al., 2008), which can eliminate the influence of different transcript lengths and sequencing discrepancies when calculating abundance (Mortazavi et al., 2008). We used the following equation:

$$
R P K M(A)=\frac{C \times 10^{6}}{\frac{N \times L}{10^{3}}}
$$

where $R P K M(A)$ is the RPKM value of transcript $A$; $C$ is the number of reads uniquely aligned to transcript $A ; N$ is the total number of fragments uniquely aligned to all transcripts; and $L$ is the number of bases in transcript $A$.

\section{Differential Expression Analysis}

Genes showing differential expression between two conditions/groups were detected using the DESeq $\mathrm{R}$ package (1.10.1), which provides statistical routines to determine differential expression from digital gene expression data using a model based on negative binomial distribution. The resulting $P$ values were adjusted using Benjamini and Hochberg's approach 


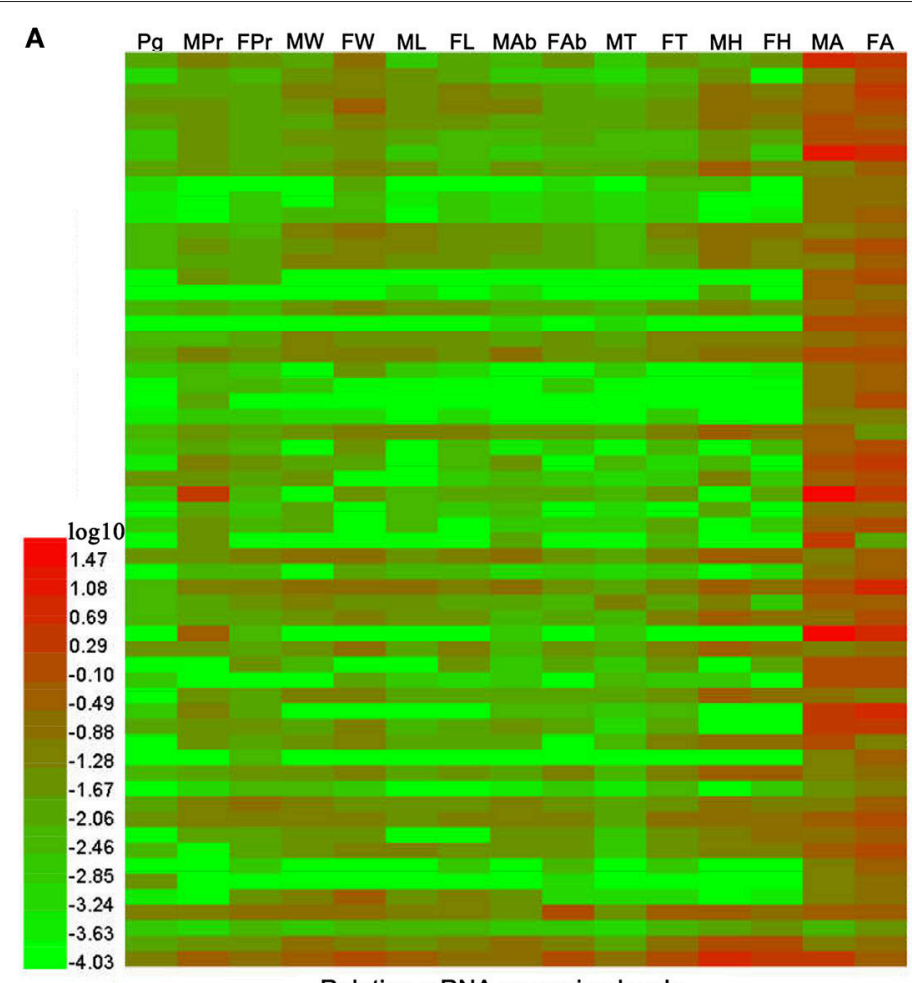

Relative mRNA expresion levels

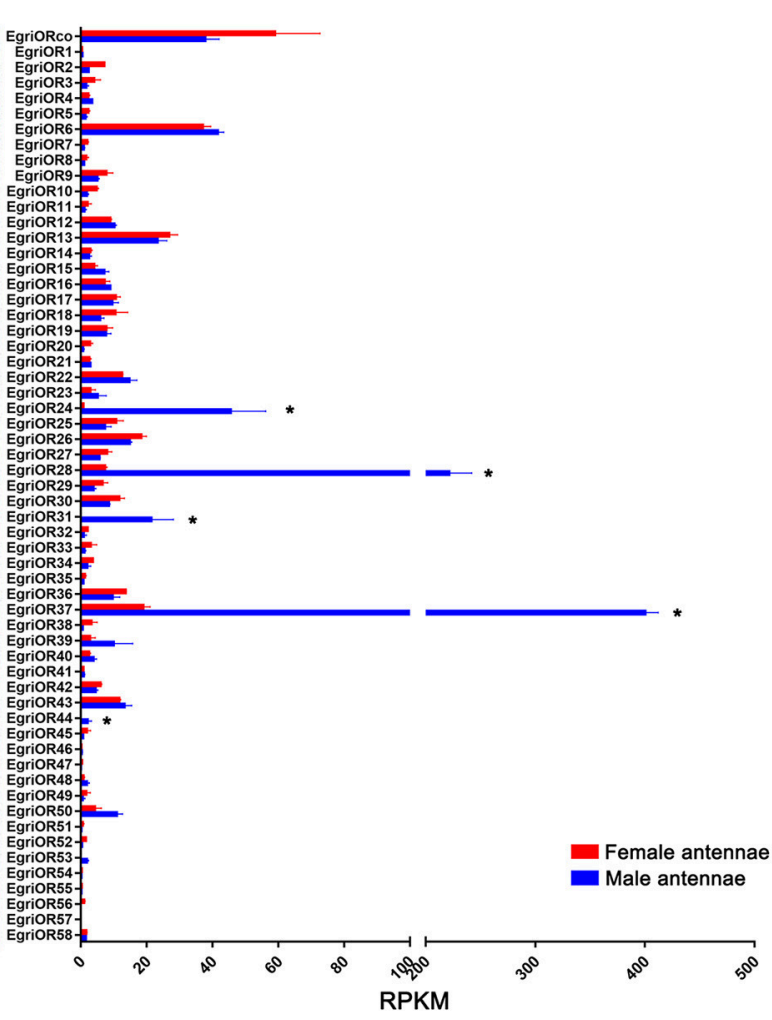

B

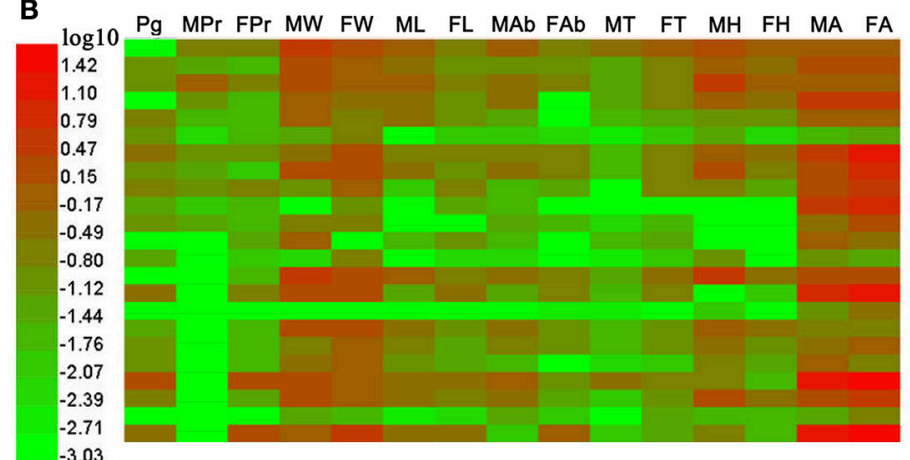

Relative mRNA expresion levels

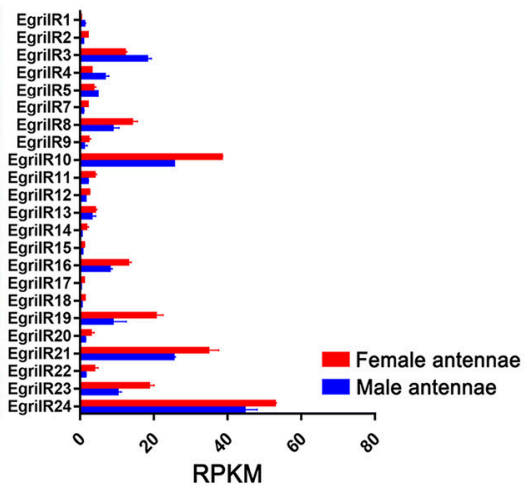

FIGURE 8 | Tissue expression profiles and abundance of selected EgriOR and EgrilR genes in antennae based on relative mRNA quantity and RPKM values. Heat map illustrates $\log _{10}$-transformed mRNA expression levels of EgriOR and EgrilR in different tissues. Histogram shows RPKM values of EgriOR and EgrilR. (A) Tissue expression profile and RPKM values of selected EgriOR genes. (B) Tissue expression profile and RPKM values of selected EgrilR genes. FA, female antennae; MA, male antennae; $\mathrm{FH}$, female head without antennae; $\mathrm{MH}$, male head without antennae; FT, female thorax; MT, male thorax; FAb, female abdomen without pheromone gland; MAb, male abdomen; FL, female legs; ML, male legs; FW, female wings; MW, male wings; FPr, female proboscis; MPr, male proboscis; Pg, pheromone gland. ${ }^{\star} P<0.05$.

to control the false discovery rate. Genes with an adjusted $P$ $<0.05$ found by DESeq were designated as being differentially expressed.

\section{Sequence Alignment and Phylogenetic Analysis}

Amino acid sequence alignments of the candidate 40 EgriOBPs and 30 EgriCSPs were performed using ClustalX 2.0 (Larkin et al., 2007), and visualized using Jalview 2.4.0 b2 (Waterhouse et al., 2009). The signal peptides of EgriOBPs and EgriCSps were predicted by SignalP 4.1 (http://www.cbs.dtu.dk/services/ SignalP/). To investigate the phylogenetic relationships of OBPs, CSPs, ORs, and IRs between E. grisescens and other typical insect species, we compared them using MAFFT (E-INS-I parameter) (Katoh and Standley, 2013). Phylogenetic trees were constructed using PhyML 3.1 with LG substitution model was used to construct a maximum likelihood phylogenetic tree using Bayesian analysis (Guindon et al., 2010). 


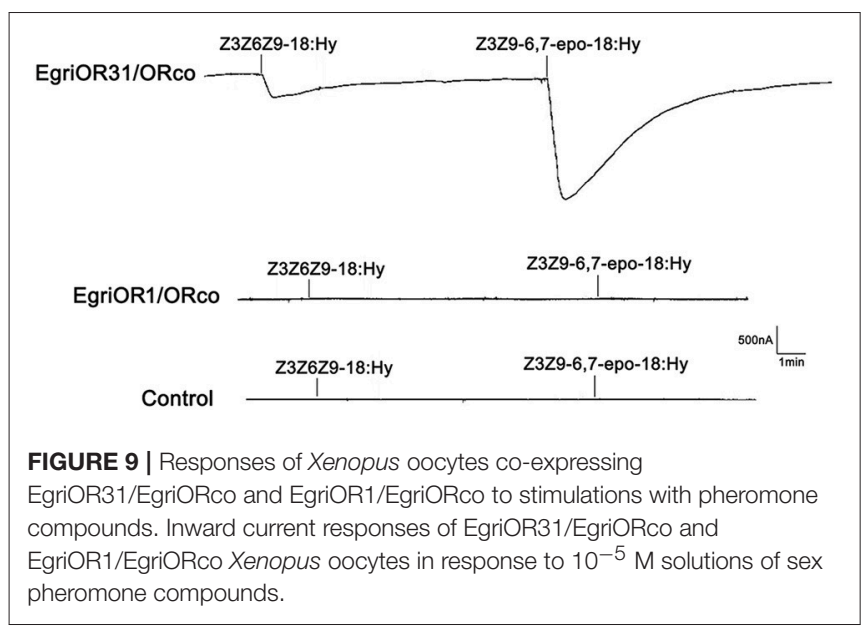

\section{Quantitative Real-Time PCR Validation}

The tissue expression patterns of $40 \mathrm{OBPs}, 30 \mathrm{CSPs}, 59 \mathrm{ORs}$, and $24 I R s$ in different tissues were measured by a $\mathrm{qPCR}$ method conducted according to the minimum information for publication of quantitative Real-Time PCR Experiments (Bustin et al., 2009). Total RNA was isolated using the SV Total Isolation System (Promega, Madison, WI, USA) according to the manufacturer's instructions. Quality of the RNA were assessed by agarose gel electrophoresis, Nanodrop (Thermo). Single-stranded cDNA templates were synthesized using the Reverse Transcription System (Promega) as per the manufacturer's instructions. The qRT-PCRs were performed on a Bio-Rad CFX96 touch real-time PCR detection system (BioRad, Hercules, CA, USA). The primers were designed using Beacon Designer 7.7 based on the E. grisescens nucleotide sequences obtained from the transcriptome data (File S2). Templates diluted into five-fold series were used to construct a relative standard curve to determine the PCR efficiencies and for quantification analysis. Each reaction was run in triplicate (technical repeat). Two reference genes, guanine nucleotidebinding protein $\mathrm{G}(\mathrm{q})$ subunit alpha and glyceraldehyde-3phosphate dehydrogenase (sequences were provided in File S3), were selected in $\mathrm{qPCR}$ analysis. A blank control without template cDNA (replacing cDNA with $\mathrm{H}_{2} \mathrm{O}$ ) served as the negative control. Each reaction included three independent biological replicates and was repeated three times (technical replicates). Relative transcript levels were calculated using the comparative $2^{-\Delta \Delta} \mathrm{Cq}$ method. The level of each tested mRNA was determined using SYBR ${ }^{\circledR}$ Premix Ex Taq $^{\text {TM }}$ II (TaKaRa, Dalian, China) according to the manufacturer's instructions.

\section{Functional Study of EgriOR31}

A Xenopus oocytes expression system was used to express the EgriOR1 and 31. EgriOR1 and 31 were amplified using specific primers (File S2). The purified PCR products were ligated into
pT7Ts vector using an In-Fusion ${ }^{\circledR}$ HD Cloning Kit (Clontech, USA) following manufacturer's instructions. The cRNAs of EgriORs were synthesized using mMESSAGE Mmachine T7 kit (Ambion, Austin, TX). Electrophysiological recording was performed according to previously reported protocols (Wang et al., 2010). Mature healthy oocytes (stage V-VII) were treated with $2 \mathrm{mg} / \mathrm{ml}$ collagenase I(GIBCO, Carlsbad, CA) in washing buffer $[96 \mathrm{mM} \mathrm{NaCl}, 2 \mathrm{mM} \mathrm{KCl}, 5 \mathrm{mM} \mathrm{MgCl}$, and $5 \mathrm{mM}$ HEPES $(\mathrm{pH}=7.6)]$ for about $1.5 \mathrm{~h}$ at room temperature. And then, oocytes were microinjected with $27.6 \mathrm{ng}$ EgriOR cRNAs and $27.6 \mathrm{ng}$ EgriORco. After injection, oocytes were incubated for $4-7$ days at $18^{\circ} \mathrm{C}$ in $1 \times$ Ringer's solution [ $96 \mathrm{mM} \mathrm{NaCl}, 2 \mathrm{mM} \mathrm{KCl}, 5 \mathrm{mM} \mathrm{MgCl}_{2}, 0.8 \mathrm{mM} \mathrm{CaCl}_{2}$, and $5 \mathrm{mM}$ HEPES $(\mathrm{pH}=7.6)]$ supplemented with $5 \%$ dialyzed horse serum, $50 \mathrm{mg} / \mathrm{ml}$ tetracycline, $100 \mathrm{mg} / \mathrm{ml}$ streptomycin, and $550 \mathrm{mg} / \mathrm{ml}$ sodium pyruvate. Whole-cell currents were recorded from the injected Xenopus oocytes with a two-electrode voltage clamp and recorded with an OC-725C oocyte clamp (Warner Instruments, Hamden, CT, USA). Stock solutions of tested compounds were prepared using ethyl alcohol, which were diluted to the indicated concentrations by $1 \times$ Ringer's buffer before use. Oocytes were exposed to $1 \times 10^{-5} \mathrm{M}$ of sex pheromone compounds and ethyl alcohol. Oocytes without cRNA injection were set as negative control. Data acquisition and analyses were carried out with Digidata 1440A and pCLAMP 10.2 software (Axon Instruments Inc., Union City, CA).

\section{AUTHOR CONTRIBUTIONS}

$\mathrm{Z}-\mathrm{QL}$ and Z-MC conceived and designed the experiments; Z-QL performed the experiments; Z-QL, Z-XL, X-MC, $\mathrm{LB}, \mathrm{Z}-\mathrm{JX}, \mathrm{YL}$, and $\mathrm{BC}$ analyzed the data; and $\mathrm{Z}-\mathrm{QL}$ wrote the manuscript. All authors reviewed the final manuscript.

\section{ACKNOWLEDGMENTS}

This study was funded by the National Natural Science Foundation of China (31701795), the Central Public-interest Scientific Institution Basal Research Fund (1610212017006), and the National Key Research \& Development (R\&D) Plan (2016YFD0200900).

\section{SUPPLEMENTARY MATERIAL}

The Supplementary Material for this article can be found online at: https://www.frontiersin.org/articles/10.3389/fphys. 2017.00953/full\#supplementary-material

File S2 | Primers used in QPCR and Vector construction.

File S3 | Sequences of guanine nucleotide-binding protein $G(q)$ subunit alpha and glyceraldehyde-3-phosphate dehydrogenase. 


\section{REFERENCES}

Ai, M., Min, S., Grosjean, Y., Leblanc, C., Bell, R., Benton, R., et al. (2010). Acid sensing by the Drosophila olfactory system. Nature 468, 691-695. doi: 10.1038/nature09537

Ai, M. R., Blais, S., Park, J. Y., Min, S., Neubert, T. A., and Suh, G. S. B. (2013). Ionotropic glutamate receptors IR64a and IR8a form a functional odorant receptor complex in vivo in Drosophila. J. Neurosci. 33, 10741-10749. doi: 10.1523/JNEUROSCI.5419-12.2013

Ando, T., Inomata, S., and Yamamoto, M. (2004). Lepidopteran sex pheromones. Top. Curr. Chem. 239, 951-961. doi: 10.1007/b95449

Angeli, S., Ceron, F., Scaloni, A., Monti, M., Monteforti, G., Minnocci, A., et al. (1999). Purification, structural characterization, cloning and immunocytochemical localization of chemoreception proteins from Schistocerca gregaria. Eur. J. Biochem. 262, 745-754. doi: 10.1046/j.1432-1327.1999.00438.x

Benton, R., Vannice, K. S., Gomez-Diaz, C., and Vosshall, L. B. (2009). Variant ionotropic glutamate receptors as chemosensory receptors in Drosophila. Cell 136, 149-162. doi: 10.1016/j.cell.2008.12.001

Bustin, S. A., Benes, V., Garson, J. A., Hellemans, J., Huggett, J., Kubista, M., et al. (2009). The MIQE guidelines: minimum information for publication of quantitative real-time PCR experiments. Clin. Chem. 55, 611-622. doi: 10.1373/clinchem.2008.112797

Cao, D., Liu, Y., Wei, J., Liao, X., Walker, W. B., Li, J., et al. (2014). Identification of candidate olfactory genes in Chilo suppressalis by antennal transcriptome analysis. Int. J. Biol. Sci. 10, 846-860. doi: 10.7150/ijbs.9297

Chang, H. T., Liu, Y., Yang, T., Pelosi, P., Dong, S. L., and Wang, G. R. (2015). Pheromone binding proteins enhance the sensitivity of olfactory receptors to sex pheromones in Chilo suppressalis. Sci. Rep. 5:13093. doi: 10.1038/srep13093

Conesa, A., Gotz, S., Garcia-Gomez, J. M., Terol, J., Talon, M., and Robles, M. (2005). Blast2GO: a universal tool for annotation, visualization and analysis in functional genomics research. Bioinformatics 21, 3674-3676. doi: 10.1093/bioinformatics/bti610

Dong, K., Sun, L., Liu, J. T., Gu, S. H., Zhou, J. J., Yang, R. N., et al. (2017). RNAiinduced electrophysiological and behavioral changes reveal two pheromone binding proteins of Helicoverpa armigera involved in the perception of the main sex pheromone component Z11-16:Ald. J. Chem. Ecol. 43, 207-214. doi: 10.1007/s10886-016-0816-6

Flower, D. R. (1996). The lipocalin protein family: structure and function. Biochem. J. 318, 1-14. doi: 10.1042/bj3180001

Forêt, S., and Maleszka, R. (2006). Function and evolution of a gene family encoding odorant binding-like proteins in a social insect, the honey bee (Apis mellifera). Genome Res. 16, 1404-1413. doi: 10.1101/gr.5075706

Gong, D. P., Zhang, H. J., Zhao, P., Xia, Q. Y., and Xiang, Z. H. (2009). The odorant binding protein gene family from the genome of silkworm, Bombyx mori. BMC Genomics 10:332. doi: 10.1186/1471-2164-10-332

González, D., Zhao, Q., McMahan, C., Velasquez, D., Haskins, W. E., Sponsel, V., et al. (2009). The major antennal chemosensory protein of red imported fire ant workers. Insect Mol. Biol. 18, 395-404. doi: 10.1111/j.1365-2583.2009.00883.x

Grabherr, M. G., Haas, B. J., Yassour, M., Levin, J. Z., Thompson, D. A., Amit, I., et al. (2011). Full-length transcriptome assembly from RNA-Seq data without a reference genome. Nat. Biotechnol. 29, 644-652. doi: 10.1038/nbt.1883

Guindon, S., Dufayard, J. F., Lefort, V., Anisimova, M., Hordijk, W., and Gascuel, O. (2010). New algorithms and methods to estimate maximum-likelihood phylogenies: assessing the performance of PhyML 3.0. Syst. Biol. 59, 307-321. doi: $10.1093 /$ sysbio/syq010

Hekmat-Scafe, D. S., Scafe, C. R., McKinney, A. J., and Tanouye, M. A. (2002). Genome-wide analysis of the odorant-binding protein gene family in Drosophila melanogaster. Genome Res. 12, 1357-1369. doi: 10.1101/gr.239402

Jiang, X. J., Guo, H., Di, C., Yu, S. L., Zhu, L. G., Huang, L. Q., et al. (2014). Sequence similarity and functional comparisons of pheromone receptor orthologs in two closely related Helicoverpa species. Insect Biochem. Mol. Biol. 48, 63-74. doi: 10.1016/j.ibmb.2014.02.010

Jin, J. Y., Li, Z. Q., Zhang, Y. N., Liu, N. Y., and Dong, S. L. (2014). Different roles suggested by sex-biased expression and pheromone binding affinity among three pheromone binding proteins in the pink rice borer, Sesamia inferens (Walker) (Lepidoptera: Noctuidae). J. Insect Physiol. 66, 71-79. doi: 10.1016/j.jinsphys.2014.05.013
Katoh, K., and Standley, D. M. (2013). MAFFT multiple sequence alignment software version 7: improvements in performance and usability. Mol. Biol. Evol. 30, 772-780. doi: 10.1093/molbev/mst010

Kulmuni, J., and Havukainen, H. (2013). Insights into the evolution of the CSP gene family through the integration of evolutionary analysis and comparative protein modeling. PLoS ONE 8:e63688. doi: 10.1371/journal.pone. 0063688

Larkin, M. A., Blackshields, G., Brown, N. P., Chenna, R., McGettigan, P. A., McWilliam, H., et al. (2007). Clustal W and Clustal X version 2.0. Bioinformatics 23, 2947-2948. doi: 10.1093/bioinformatics/btm404

Leal, W. S. (2012). Odorant reception in insects: roles of receptors,binding proteins, and degrading enzymes. Annu. Rev. Entomol. 58, 373-463. doi: 10.1146/annurev-ento-120811-153635

Li, Z. Q., Zhang, S., Luo, J. Y., Cui, J. J., Ma, Y., and Dong, S. L. (2013). Two Minus$\mathrm{C}$ odorant binding proteins from Helicoverpa armigera display higher ligand binding affinity at acidic $\mathrm{pH}$ than neutral pH. J. Insect Physiol. 59, 263-272. doi: 10.1016/j.jinsphys.2012.12.004

Li, Z. Q., Zhang, S., Luo, J. Y., Zhu, J., Cui, J. J., and Dong, S. L. (2015). Expression analysis and binding assays in the chemosensory protein gene family indicate multiple roles in Helicoverpa armigera. J. Chem. Ecol. 41, 473-485. doi: 10.1007/s10886-015-0574-X

Liu, C., Liu, Y., Walker, W. B., Dong, S., and Wang, G. (2013). Identification and functional characterization of sex pheromone receptors in beet armyworm Spodoptera exigua (Hubner). Insect Biochem. Mol. Biol. 43, 747-754. doi: 10.1016/j.ibmb.2013.05.009

Liu, N. Y., Liu, C. C., and Dong, S. L. (2013). Functional differentiation of pheromone-binding proteins in the common cutworm Spodoptera litura. Comp. Biochem. Physiol. A Mol. Integr. Physiol. 165, 254-262. doi: 10.1016/j.cbpa.2013.03.016

Liu, N. Y., Yang, F., Yang, K., He, P., Niu, X. H., Xu, W., et al. (2014). Two subclasses of odorant-binding proteins in Spodoptera exigua display structural conservation and functional divergence. Insect Mol. Biol. 24, 167-182. doi: 10.1111/imb.12143

Liu, N. Y., Yang, K., Liu, Y., Xu, W., Anderson, A., and Dong, S. L. (2015). Two general-odorant binding proteins in Spodoptera litura are differentially tuned to sex pheromones and plant odorants. Comp. Biochem. Physiol A Mol. Integr. Physiol. 180, 23-31. doi: 10.1016/j.cbpa.2014.11.005

Liu, Y., Gu, S., Zhang, Y., Guo, Y., and Wang, G. (2012). Candidate olfaction genes identified within the Helicoverpa armigera antennal transcriptome. PLoS ONE 7:e48260. doi: 10.1371/journal.pone.0048260

Ma, T., Xiao, Q., Yu, Y. G., Wang, C., Zhu, C. Q., Sun, Z. H., et al. (2016). Analysis of tea geometrid (Ectropis grisescens) pheromone gland extracts using GC-EAD and GCxGC/TOFMS. J. Agric. Food Chem. 64, 3161-3166. doi: 10.1021/acs.jafc.6b00339

Maleszka, J., Foret, S., Saint, R., and Maleszka, R. (2007). RNAi-induced phenotypes suggest a novel role for a chemosensory protein CSP5 in the development of embryonic integument in the honeybee (Apis mellifera). Dev. Genes Evol. 217, 189-196. doi: 10.1007/s00427-006-0127-y

Millar, J. G. (2000). Polyene hydrocarbons and epoxides: a second major class of lepidopteran sex attractant pheromones. Annu. Rev. Entomol. 45, 575-604. doi: 10.1146/annurev.ento.45.1.575

Mortazavi, A., Williams, B. A., McCue, K., Schaeffer, L., and Wold, B. (2008). Mapping and quantifying mammalian transcriptomes by RNA-Seq. Nat. Methods 5, 621-628. doi: 10.1038/nmeth.1226

Nakagawa, T., Pellegrino, M., Sato, K., Vosshall, L. B., and Touhara, K. (2012). Amino acid residues contributing to function of the heteromeric insect olfactory receptor complex. PLoS ONE 7:e32372. doi: 10.1371/journal.pone.0032372

Pelosi, P., Iovinella, I., Felicioli, A., and Dani, F. R. (2014). Soluble proteins of chemical communication: an overview across arthropods. Front. Physiol. 5:320. doi: 10.3389/fphys.2014.00320

Rytz, R., Croset, V., and Benton, R. (2013). Ionotropic receptors (IRs): chemosensory ionotropic glutamate receptors in Drosophila and beyond. Insect Biochem. Mol. Biol. 43, 888-897. doi: 10.1016/j.ibmb.2013.02.007

Sanes, J. T., and Plettner, E. (2016). Gypsy moth pheromone-binding protein-ligand interactions: $\mathrm{pH}$ profiles and simulations as tools for detecting polar interactions. Arch. Biochem. Biophys. 606, 53-63. doi: 10.1016/j.abb.2016.07.008 
Spinelli, S., Lagarde, A., Iovinella, I., Legrand, P., Tegoni, M., Pelosi, P., et al. (2012). Crystal structure of Apis mellifera OBP14, a C-minus odorant-binding protein, and its complexes with odorant molecules. Insect Biochem. Mol. Biol. 42, 41-50. doi: 10.1016/j.ibmb.2011.10.005

Spletter, M. L., and Luo, L. Q. (2009). A new family of odorant receptors in Drosophila. Cell 136, 23-25. doi: 10.1016/j.cell.2008.12.031

Sun, M. J., Liu, Y., Walker, W. B., Liu, C. C., Lin, K. J., Gu, S. H., et al. (2013). Identification and characterization of pheromone receptors and interplay between receptors and pheromone binding proteins in the diamondback moth, Plutella xyllostella. PLoS ONE 8:e62098 . doi: 10.1371/journal.pone.0062098

Sun, X. L., Wang, G. C., Gao, Y., Zhang, X. Z., Xin, Z. J., and Chen, Z. M. (2014). Volatiles emitted from tea plants infested by Ectropis obliqua larvae are attractive to conspecific moths. J. Chem. Ecol. 40, 1080-1089. doi: 10.1007/s10886-014-0502-5

Sun, Y. L., Huang, L. Q., Pelosi, P., and Wang, C. Z. (2012). Expression in antennae and reproductive organs suggests a dual role of an odorantbinding protein in two sibling Helicoverpa species. PLoS ONE 7:e30040. doi: 10.1371/journal.pone.0030040

Tegoni, M., Campanacci, V., and Cambillau, C. (2004). Structural aspects of sexual attraction and chemical communication in insects. Trends Biochem. Sci. 29, 257-264. doi: 10.1016/j.tibs.2004.03.003

Wang, G., Carey, A. F., Carlson, J. R., and Zwiebel, L. J. (2010). Molecular basis of odor coding in the malaria vector mosquito Anopheles gambiae. Proc. Natl. Acad. Sci. U.S.A. 107, 4418-4423. doi: 10.1073/pnas.0913392107

Waterhouse, A. M., Procter, J. B., Martin, D. M., Clamp, M., and Barton, G. J. (2009). Jalview Version 2-a multiple sequence alignment editor and analysis workbench. Bioinformatics 25, 1189-1191. doi: 10.1093/bioinformatics/btp033

Ye, J., Fang, L., Zheng, H., Zhang, Y., Chen, J., Zhang, Z., et al. (2006). WEGO: a web tool for plotting GO annotations. Nucleic Acids Res. 34, W293-W297. doi: $10.1093 /$ nar/gkl031

Zhang, D. D., Wang, H. L., Schultze, A., Fross, H., Francke, W., Krieger, J., et al. (2016). Receptor for detection of a Type II sex pheromone in the winter moth Operophtera brumata. Sci. Rep. 6:18576. doi: 10.1038/srep18576
Zhang, Y. L. V., Ni, J. F., and Montell, C. (2013). The molecular basis for attractive salt-taste coding in Drosophila. Science 340, 1334-1338. doi: 10.1126/science.1234133

Zhang, Y. N., Ye, Z. F., Yang, K., and Dong, S. L. (2014a). Antennapredominant and male-biased CSP19 of Sesamia inferens is able to bind the female sex pheromones and host plant volatiles. Gene 536, 279-286. doi: 10.1016/j.gene.2013.12.011

Zhang, Y. N., Zhang, J., Yan, S. W., Chang, H. T., Liu, Y., Wang, G. R., et al. (2014b). Functional characterization of sex pheromone receptors in the purple stem borer, Sesamia inferens (Walker). Insect Mol. Biol. 23, 611-620. doi: 10.1111/imb.12109

Zhou, J. J. (2010). Odorant-binding proteins in insects. Vitam. Horm. 83, 241-272. doi: 10.1016/S0083-6729(10)83010-9

Zhou, J. J., Huang, W., Zhang, G. A., Pickett, J. A., and Field, L. M. (2004). "Plus-C" odorant-binding protein genes in two Drosophila species and the malaria mosquito Anopheles gambiae. Gene 327, 117-129. doi: 10.1016/j.gene.2003.11.007

Zhou, J. J., Robertson, G., He, X. L., Dufour, S., Hooper, A. M., Pickett, J. A., et al. (2009). Characterisation of Bombyx mori odorant-binding proteins reveals that a general odorant-binding protein discriminates between sex pheromone components. J. Mol. Biol. 389, 529-545. doi: 10.1016/j.jmb.2009. 04.015

Conflict of Interest Statement: The authors declare that the research was conducted in the absence of any commercial or financial relationships that could be construed as a potential conflict of interest.

Copyright () $2017 \mathrm{Li}, \mathrm{Luo}, \mathrm{Cai}, \mathrm{Bian}, \mathrm{Xin}, \mathrm{Liu}, \mathrm{Chu}$ and Chen. This is an open-access article distributed under the terms of the Creative Commons Attribution License (CC $B Y$ ). The use, distribution or reproduction in other forums is permitted, provided the original author(s) or licensor are credited and that the original publication in this journal is cited, in accordance with accepted academic practice. No use, distribution or reproduction is permitted which does not comply with these terms. 